\title{
Infestation massive des Ephemera vulgata du Manaurie (près Les Eyzies), par Spirinella adipopbila (Protozoa.) n.g., n.sp.
}

\author{
Par Lucie ARVY et Bernadette DELAGE
}

L'étude histoenzymologique des tubes de Malpighi entreprise par l'une de nous (1), nous a conduites à récolter des larves d'Insectes aquatiques et plus particulièrement des larves d'Ephéméroptères. Rayonnant autour de la station biologique des Eyzies, nous avons examiné des Ephémères récoltées dans la Beune et divers ruisselets: Font-deGaume et autres qui s'y déversent, sans noter d'anomalies; par contre, les Ephemera vulgata récoltées dans le Manaurie étaient, sans exception, infestées d'une manière massive (fig. 1) par un parasite qui a retenu notre attention par son étrangeté et son abondance. L'animal, dans son ensemble (fig. 1 à 4), est discoïde, non encapsulé, ni enkysté ; il paraît libre, mêlé aux leucocytes dans toute la cavité générale de l'éphémère et plus particulièrement dans le tissu adipeux. Chaque disque est en réalité formé d'environ huit tours de spire enroulés les uns sur les autres, un peu comme les spires de Mollusques Planorbidés, à ceci près que les divers tours de spire ont tous la même épaisseur. Il suffit d'une légère pression, sur la lamelle d'une préparation montée, pour voir les spires se dérouler (fig. 3) en un long ruban de largeur uniforme, jusqu'à l'extrémité arrondie, qui était au centre de la spire. L'animal est placé là où serait l'opercule d'un Planorbide et toute la spire n'est qu'un long appendice tubuliforme et rubanné. L'extrémité du ruban qui contient l'animal se termine à angle droit. Le ruban ne porte aucune striation, ni granulation; il s'est révélé être chromophobe vis-à-vis de tous les colorants que nous avons pu mettre en œuvre ; il s'agit indubitablement d'un appendice, utilisé lors de la natation, ou de la fixation du parasite sur son hôte, ou sur quelque support adéquat, quand il est libre ; sa longueur varie entre 600 et $700 \mu$, sa largeur est de $15 \mu$, et il ne dépasse pas $1 \mu$ d'épaisseur (fig. 3).

Le parasite proprement dit est situé dans une sorte de chambre ovoïde (de $12 \mu$ de long et $8 \mu$ de large), très aplatie, abritée dans l'appendice tubuliforme ; il a parfois la forme d'un ovoïde aplati $(9 \mu \times 6 \mu)$ uniformément et finement granuleux, sauf au niveau du noyau, qui apparaît en clair ; mais, parfois il est différencié (fig. 5 à 8), en un organite vermiforme, replié sur lui-même en $\mathrm{V}$, en $\mathrm{U}$, ou en $\mathrm{O}$; un fin canal fait communiquer son extrémité antérieure effilée avec l'extérieur (fig. 6 et 7), qu'il 
affronte au niveau d'une légère dépression du tube; il semble être uninucléé ; son cytoplasme se colore uniformément par le rouge neutre, en rose pâle avec quelques fins granules rouge-foncé, plus particulièrement nombreux autour du noyau; le bleu de crésyle brillant colore électivement son cytoplasme en bleu clair.

Lorsque l'infestation est légère, les parasites sont cantonnés à proximité immédiate du tube digestif ; mais, quand l'infestation est très forte, les parasites occupent tout l'espace réservé au tissu adipeux chez un Ephémère normal; on les trouve aussi dans la gonade; dans ces cas d'infestations massives, le tissu adipeux n'existe plus qu'à l'état de trace. Le parasite ne semble exercer aucune action toxique sur son hôte; les tissus de l'Ephémère paraissent le tolérer parfaitement; aucun pullulement de leucocytes, ni réaction conjonctive n'est visible à son voisignage. L'infestation même massive ne s'oppose pas à la mue imaginale, car des larves appartenant à des gîtes infestés à $100 \%$ muent et, preuve supplémentaire, les imagos issues de ces larves contiennent le parasite.

\section{Discussion}

Le parasite spiralé des larves d'Ephemera vulgata L. est indubitablement le même que celui qui a été vu par Vayssière (3) chez des Oligoneuria garumnica; les croquis du mémoire de cet auteur permettent de l'affirmer. Cependant, aucun caractère n'incite à le rattacher aux Cestodes comme l'admettait Vayssière. La forme générale du parasite rappelle d'assez près celle des Helicosporidia, mais, il ne s'agit apparemment que d'une convergence de forme ; en effet, chez la seule espèce bien étudiée, Helicosporidium parasiticum (Keilin, 1921), la spore mûre est complexe, l'organisme parasitaire est toujours inclus dans un sac membraneux et il est indépendant de l'élatère, différenciation qui assure sa dispersion; chez Spirinella adipophila, l'organisme parasitaire est solidaire d'un ruban dans lequel il s'abrite. En outre, les rapports de taille sont entièrement différents : la spore de Helicosporidium parasiticum ne dépasse pas $6 \mu$ de diamètre, alors que les parasites enroulés d'Ephemera vulgata atteignent $60 \mu$.

Les antécédents et le devenir de ce parasite nous sont inconnus; tous les essais que nous avons tentés pour le modifier ou le voir évoluer ont échoué.

Ce type de parasitisme chez les Ephéméroptères ne doit pas être très fréquent, car l'une de nous a revu de nombreuses coupes d'Ephéméroptères faites entre 1951 et 1962 , en collaboration avec M. Gabe, en vue d'autres recherches, sans en trouver trace ; c'est pourquoi, nous le décrivons et le dénommons Spirinella adipophila. Il semble être spécifique de certains Ephéméroptères car, dans un gîte infesté, donné, les larves cohabitantes variées (Odonates, Plécoptères, Coléoptères) n'ont jamais présenté ce genre de parasitisme.

Jusqu'à plus ample informé, nous plaçons ce parasite parmi les Grégarinidés, sinon à leur voisinage immédiat, et nos recherches actuelles sont orientées vers un éventuel apparentement de Spirinella adipophila avec les Grégarines du tube digestif d'Ephemera vulgata. 

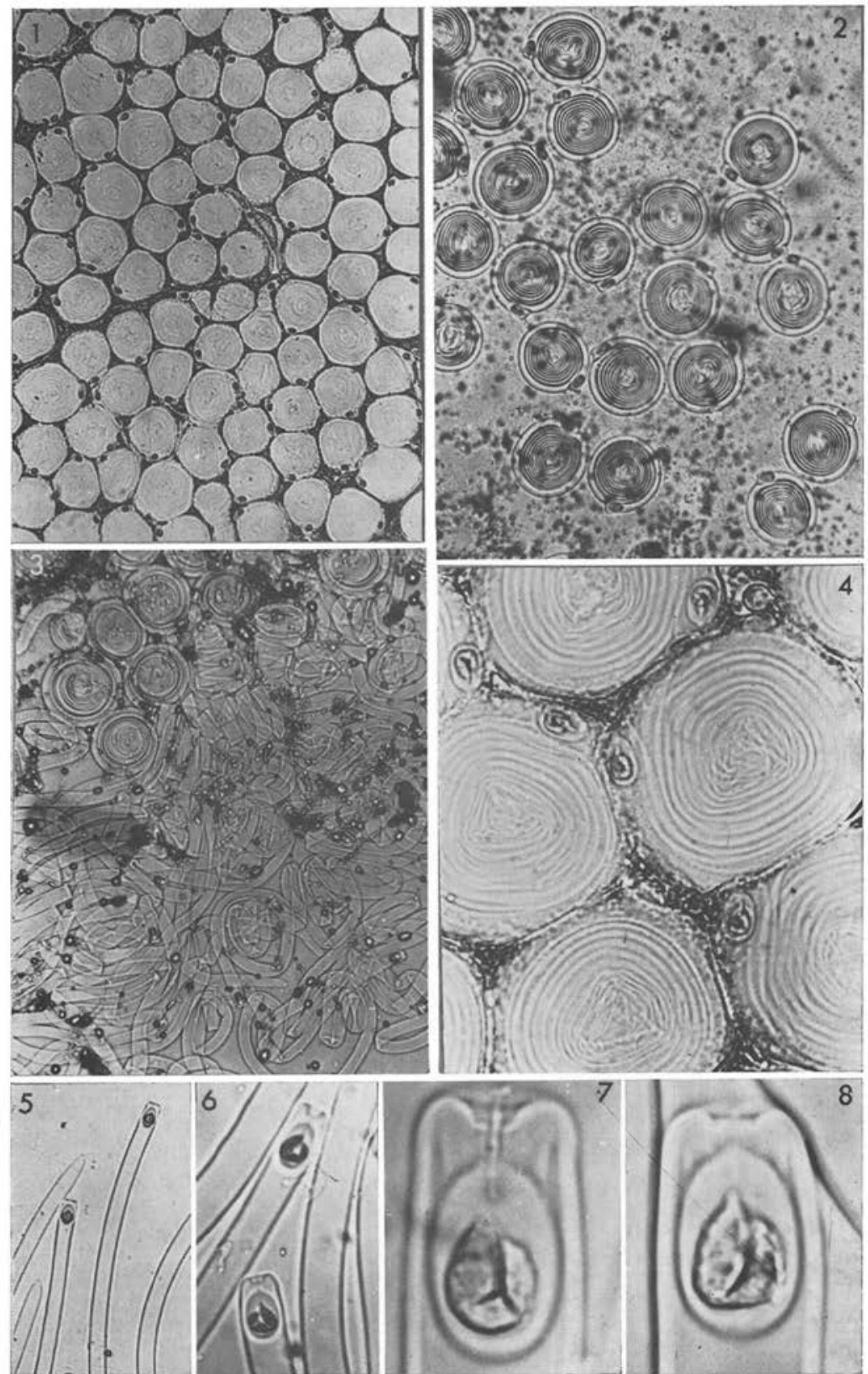

FIG. 1-2 et 4. - Spirinella adipophila, nov. gen., nov. sp. FIg. 2 : Vu à frais. Fig. 1 et 4: Vus à deux grossissements différents, après coloration par le rouge neutre. FIG. 3: Déroulement des spires, provoqué par une légère pression. Fıg. 5: Les deux extrémités du ruban. FIG. 5, 6, 7 et 8: Aspects du parasite dans sa chambre 


\section{Bibliographie}

1. ARVy (L.), 1963. - C.R. Acad Sci., 256, 5439.

2. - et Gabe (M.), 1952. - Experientia, 8, 12; 1952, Bull. Soc. zool. Fr., 77, 229 ; 1952, La Cellule, 55, 203-22 ; 1954, Pubbl. Stat. zool. Napoli ; 1953, suppl. 24, 54 ;

Zeit. Zellf., 1962, 38, 591-610; Mémoire Soc. Endoc., $\mathrm{n}^{\circ}$ 12, 331-44.

3. VAyssière (A.), 1882. - Recherches sur l'organisation des larves des Ephémères, Thèse Fac. Sci., Paris.

(C.N.R.Z. à Jouy-en-Josas-78, et Station biologique des Eyzies-24) 